\title{
Changes in Aging Mouse Neuromuscular Junctions Are Explained by Degeneration and Regeneration of Muscle Fiber Segments at the Synapse
}

\author{
Yue Li, Young il Lee, and Wesley J. Thompson \\ Section of Molecular Cell and Developmental Biology, School of Biological Sciences and Institutes for Cell and Molecular Biology and Neuroscience, \\ University of Texas at Austin, Austin, Texas 78712
}

\begin{abstract}
Vertebrate neuromuscular junctions are highly stable synapses, retaining the morphology they achieve in early postnatal development throughout most of life. However, these synapses undergo dramatic change during aging. The acetylcholine receptors (AChRs) change from smooth gutters into fragmented islands, and the nerve terminals change similarly to be varicosities apposed to these islands. These changes have been attributed to a slow deterioration in mechanisms maintaining the synapse. We have used repeated, vital imaging to investigate how these changes occur in the sternomastoid muscle of aging mice. We have found, contrary to expectation, that individual junctions change infrequently, but change, when it occurs, is sudden and dramatic. The change mimics that reported previously for cases in which muscle fibers are deliberately damaged: most of the AChRs present disappear rapidly and are replaced by a new set of receptors that become fragmented. The fiber segment underneath the synapse has centrally located nuclei, showing that this segment has undergone necrosis, quickly regenerated, and been reinnervated with an altered synapse. We show that necrotic events are common in aged muscle and have likely been missed previously as a cause of the alterations in aging because central nuclei are a transient phenomenon and the necrotic events at the junction infrequent. However, the changes are permanent and accumulate over time. Interventions to reduce the neuromuscular changes during aging should likely focus on making muscle fibers resistant to injury.
\end{abstract}

\section{Introduction}

The vertebrate neuromuscular junction (NMJ) is formed by the terminal branches of a motor neuron apposed to accumulations of acetylcholine receptors (AChRs) in the membrane near the center of each muscle fiber. Early in postnatal development, each NMJ achieves its unique "pretzel" shape given by the pattern of these terminal branches and AChRs (Marques et al., 2000; Walsh and Lichtman, 2003). In mice, this structure is remarkably stable throughout most of life (Lichtman et al., 1987), except for intercalary growth (and shrinkage) as muscle fibers and the synaptic site change in size (Balice-Gordon and Lichtman, 1990; BaliceGordon et al., 1990). However, this structure becomes unstable during the events of aging (Gutmann et al., 1971; Courtney and Steinbach, 1981; Balice-Gordon, 1997; Valdez et al., 2010). Previous studies suggest a gradual loss of synaptic area as the AChRs fragment into small islands and the nerve terminal develops varicosities (Gutmann et al., 1971; Courtney and Steinbach, 1981;

Received July 13, 2011; revised Aug. 19, 2011; accepted Aug. 23, 2011.

Author contributions:Y.L., Y.i.L., and W.J.T. designed research;Y.L. and Y.i.L. performed research; Y.L., Y.i.L., and W.J.T. analyzed data; Y.L., Y.i.L., and W.J.T. wrote the paper.

This work was supported by NIH Grant NS20480. We thank N. Priebe, T. Taub-Montemayor, J. Morgan, and M. Mikesh for discussions and for comments on this manuscript. We thank Dr. Yi Zuo for the kind gift of aged transgenic mice from her colony.

The authors declare no competing financial interests.

Correspondence should be addressed to Wesley J. Thompson, Section MCDB, University of Texas, 1 University Station C1000, Austin, TX 78712. E-mail: wes@mail.utexas.edu.

DOI:10.1523/JNEUROSCI.3590-11.2011

Copyright $\odot 2011$ the authors $\quad 0270-6474 / 11 / 3114910-10 \$ 15.00 / 0$
Balice-Gordon, 1997; Wood and Slater, 2001; Valdez et al., 2010) (see, for example, Fig. 1). We have conducted vital imaging of the sternomastoid muscle in aging mice in an attempt to visualize how these changes occur over time. We find, like others, that older muscles contain a preponderance of junctions that have the aged phenotype but also have a smaller fraction that have the morphology of younger junctions. Surprisingly, both sets of junctions are usually remarkably stable. However, we find that individual junctions of both phenotypes undergo rapid, abrupt changes. The changes in these synapses mirror those that occur upon deliberate damage to the postsynaptic muscle fiber (Duchen et al., 1974; Couteaux et al., 1988; Rich and Lichtman, 1989; Li and Thompson, 2011). The AChRs at these junctions rapidly disappear as the muscle fiber underneath them undergoes necrosis. This muscle fiber segment quickly regenerates and can be identified by the unique location of its nuclei in the center of the fiber cross section. This segment is quickly innervated by the nerve terminal that remains nearby (Rich and Lichtman, 1989; Li and Thompson, 2011). However, the new synapse is significantly altered with respect to the previous one. Similar changes occur in the mouse model of Duchenne muscular dystrophy in which fibers degenerate and regenerate (Lyons and Slater, 1991) and may be present in other neuromuscular pathologies as well. Our findings provide strong evidence that fiber injury and degeneration explain major aspects of the aging of these synapses in the mouse. These injury events occur randomly along muscle fibers, occasionally involving the segment underneath the NMJ, and when they do, result in alterations in this contact. Although the 


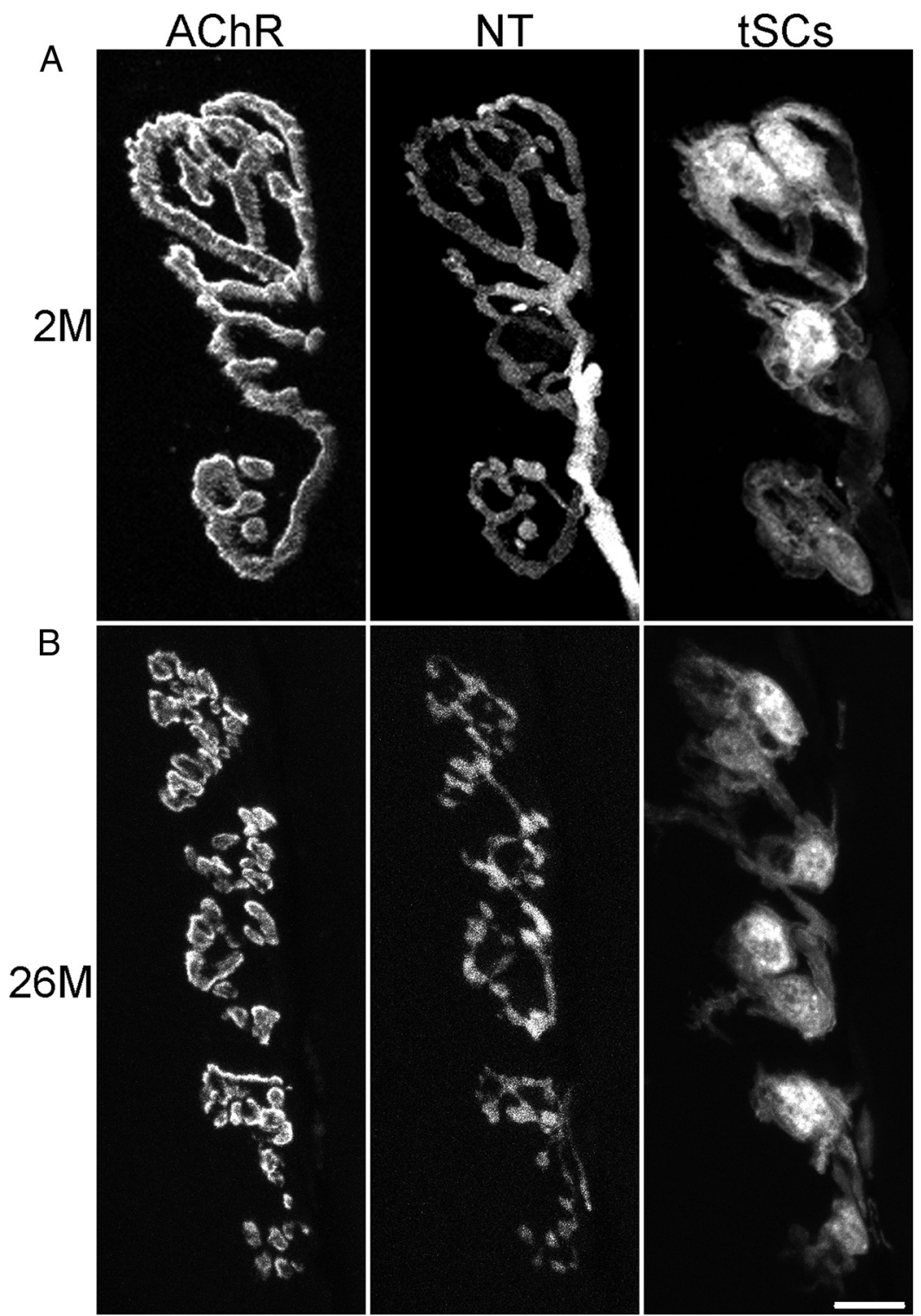

Figure 1. Aging produces striking morphological changes in NMJs of the mouse sternomastoid muscle. Confocal images of a young ( 2 months of age) NMJ ( $\boldsymbol{A}$ ) and an old, fragmented NMJ ( $\boldsymbol{B}$ ) (bottom panels; 26 months of age). In both cases, a motor nerve terminal (NT) is present on the surface of the muscle fiber, formed by branches of a single motor axon. In the young junction $(\boldsymbol{A})$, the NT branches form smooth contacts with the muscle fiber surface where a high concentration of AChRs are accumulated into largely continuous gutters, giving the appearance of a pretzel. The synapse has a small number of glial cells (tSCs) that cover the branches of the NT. In contrast, the NMJ in the old animal $(\boldsymbol{B})$ has AChRs that are fragmented into small islands that are each apposed by a varicosity in the NT; varicosities in the NT are commonly linked by thin neurites. The $\mathrm{SSC}$ appear more active and are commonly seen to be extending short processes to the sides of the NT. NT and SCs were labeled by transgenic expression of fluorescent proteins (CFP and GFP, respectively), and AChRs with a subsaturating concentration of Alexa 647 bungarotoxin. Scale bar, $10 \mu \mathrm{m}$.

individual events appear sudden and random, their consequences accumulate in the muscle gradually over time.

\section{Materials and Methods}

Mice were, with a few exceptions, transgenic for S100-green fluorescent protein (GFP) (Zuo et al., 2004) and thyl-cyan fluorescent protein (CFP) (Feng et al., 2000) and of both sexes. The S100-GFP mice were originally generated in $\mathrm{DB} 9 \times \mathrm{F}_{6}$ hybrids but have been extensively backcrossed to C57BL/6 for many generations within the animal colony at the University of Texas. Mice were fed a standard diet ad libitum and housed in standard $10.5 \times 6.5 \times 5$ inch mouse cages. Procedures for vital imaging have been extensively described previously (Zuo et al., 2004; Li and Thompson, 2011) and are presented only in outline form here. Mice were anesthetized with ketamine-xylazine $(0.10-0.15 \mathrm{ml}$ of a $0.9 \% \mathrm{NaCl}$ so- lution containing $17.4 \mathrm{mg} / \mathrm{ml}$ ketamine and 2.6 $\mathrm{mg} / \mathrm{ml}$ xylazine, initial dose) and were given an additional dose (one-quarter to one-half of the initial dose) during the imaging session if they developed reflexive response to a pinch of the hindfoot. The mouse was secured to the stage of a microscope in the supine position with rubber bands attached to the forelimbs and the incisors. The mouse was intubated via an oral tube and its respiratory movements driven from a small animal ventilator. Aseptic surgery was performed to expose, by midline incision in the neck, the sternomastoid muscle. A small metal platform was maneuvered underneath the muscle and the muscle elevated slightly using a micromanipulator. The top of the muscle was compressed slightly against this metal platform to flatten it by a wire hoop also micromanipulated into place. Bungarotoxin, $2 \mu \mathrm{g} / \mathrm{ml}$ in sterile lactated Ringer's, was applied to the muscle for 5 min to label AChRs. This dose labels $<30 \%$ of the receptors, far less than the $70 \%$ blockade required to lower the safety factor so that transmission is ineffective (Lingle and Steinbach, 1988). For the vital imaging experiments, we found, as reported previously (Balice-Gordon, 1997), that rhodamine-bungarotoxin gave labeling with high background in aged animals, likely because of autofluorescence in the red channel from contents of the muscle fibers, reported to be lipofuscin. We therefore used Alexa 647 bungarotoxin in which channel this autofluorescence was far less prominent. We found that, in terminal experiments, the autofluorescence disappeared following fixation, so that rhodamine bungarotoxin could be used as a second label here. Vital images were collected from an upright epifluorescence microscope (Leica DMR) using a Nikon $40 \times, 0.8 \mathrm{NA}$ water-immersion lens and captured with a cooled CCD camera (Hamamatsu Orca) using iVision software and a Macintosh computer. During image capture, the respirator was briefly turned off to allow the acquisition of images during a short period in which the animal was not making respiratory movements on its own. After surgery, the wound was closed with suture and the animal monitored during recovery under a heat lamp. When the animal was fully recovered and eating and drinking on its own, it was returned to its home cage.

Procedures for the laser ablation study are fully described (van Mier and Lichtman, 1994; Li and Thompson, 2011). Briefly, the beam from a nitrogen-pumped, coumarin dye laser was focused in specimen plane of the microscope and applied to a muscle fiber at three to four sites on both sides of the bungarotoxin-labeled NMJ. This produced ablation of the fiber segment underneath the NMJ without damaging the junction itself. Results demonstrating the effectiveness of the ablation method, the disappearance of the previous population of AChRs, the time course of regeneration of the fiber and the appearance of new AChRs, the status of the basal lamina, and the status of the nerve terminal during the period of fiber absence and during regeneration of the fiber are presented in the study by Li and Thompson (2011).

In the vital imaging experiments, we reidentified the same NMJ by capturing an image of its position within the $10 \times$ field of view in the microscope relative to the surrounding NMJs in the endplate band during the collection of the first image. This map was then used in the 
subsequent imaging sessions to locate the junction of interest in the field of view of a $40 \times$ objective. The distinctive, unique pretzel pattern to the bungarotoxin-labeled AChRs in each of the surrounding NMJs served as fiducial markers. Furthermore, the pattern of nerve branching and SCs in the CFP channel and GFP channels, and the position of nerve entry into the junction could also be used as identifying criteria. Occasionally, the junctions changed their orientation so they no longer presented en face (Li and Thompson, 2011). In such cases, we considered the junction to be the original one only if the surrounding NMJs gave us absolute confidence of its identity.

In some experiments, we used a reagent designed to preserve mRNA in the tissue samples (Ambion RNAlater). In these cases, we perfused animals killed with phenobarbital $(0.2 \mathrm{ml}$ of $390 \mathrm{mg} / \mathrm{ml})$ through the left ventricle with this solution. Following dissection, the muscles were fixed in $4 \%$ paraformaldehyde and processed as described previously ( $\mathrm{Li}$ and Thompson, 2011).

For the two-color experiments, an animal was anesthetized as above but was not intubated. The sternomastoid was exposed, but no platforms were placed around it. A solution of Alexa 647 bungarotoxin of the concentration above was applied to the surface of the muscle for $5 \mathrm{~min}$, the muscle was rinsed thoroughly with sterile lactated Ringer's, and the wound was closed.

For terminal experiments, mice were killed with phenobarbital (see above). Muscles were dissected, pinned to Sylgard-lined dishes at roughly resting length, and fixed for $30 \mathrm{~min}$ with $4 \%$ phosphate-buffered paraformaldehyde. Following rinsing with PBS, additional bungarotoxin labeling and initial DAPI labeling $\left(10^{-4} \mathrm{mg} / \mathrm{ml}\right)$ was performed by $30 \mathrm{~min}$ incubations following by rinsing with PBS. A small group of fibers, including for the vital imaging experiments, those NMJs that had been imaged in the animal, was dissected from the surface of the muscle and mounted in fluorescence mounting medium under a coverslip on a glass slide. The NMJs were then imaged using a Leica DMR microscope with $63 \times, 1.4$ NA lens or with a confocal microscope (Leica SP5) with an identical objective. For wide-field microscopy, images of various focal depths were combined to produce a montage, or, in the case of confocal images, $z$-stacks were collected and manipulated via software. For the vital imaging experiments, the same junctions imaged vitally were located and imaged in the dissected muscle based on the unique morphology of their AChRs and the AChRs on the surrounding fibers. To identify nuclei that were truly central, the levator auris muscle was labeled with an antibody to dystrophin (Abcam; ab 15277; used at 1:300) and a second antibody (Alexa 647-goat anti-mouse; 1:250), enabling the membranes of the muscle fibers to be visualized, or, in the case of the sternomastoid muscles, the gain on the confocal photomultiplier tube was turned up so that the background fluorescence of the components of the fiber in the rhodamine channel could be used to identify the extent of the fiber in the $z$-sections.

Data are reported as mean \pm SEM. All animal procedures were approved by the University of Texas Institutional Animal Care and Use Committee.

\section{Results}

NMJs in the sternomastoid muscles of mice exhibit profound changes as the animals age

Our experiments examining aging NMJs used vital imaging in a muscle in the neck, the sternomastoid. This muscle offers particular advantages for this type of study (Lichtman et al., 1987; van Mier et al., 1994). To verify in a quantitative fashion that the sternomastoid undergoes the same kind of changes reported recently for hindlimb muscles (Valdez et al., 2010) and to obtain a time course for these changes, we examined sternomastoid muscles of mice aged 3 weeks to 26 months. These C57BL/6 animals were transgenic for thy1-CFP (Feng et al., 2000), resulting in blue fluorescent motor axons and transgenic for S100-GFP (Zuo et al., 2004), resulting in green fluorescent Schwann cells. In addition, we applied rhodamineconjugated bungarotoxin to examine the AChRs and DAPI to label nuclei. The muscles were examined in whole mount.

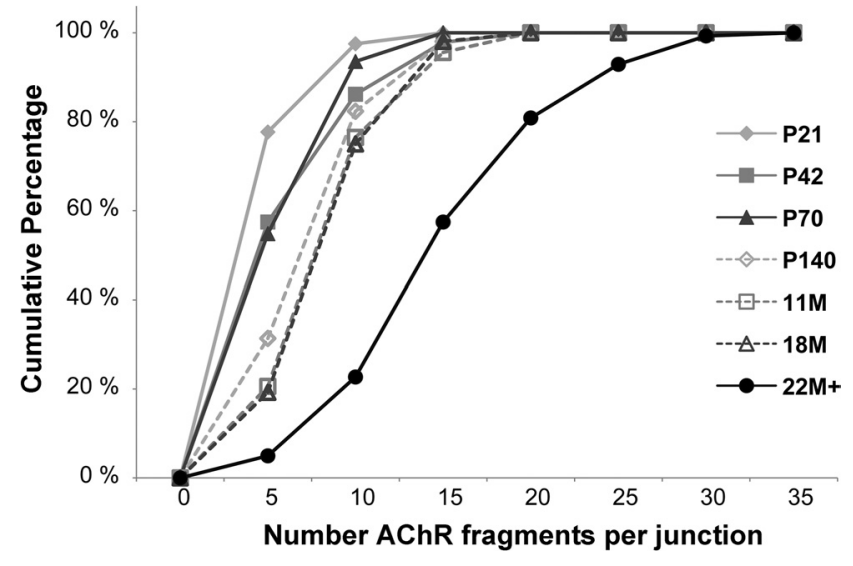

Figure 2. The number of AChR fragments in NMJs of the mouse sternomastoid increases dramatically as the animals age. Cumulative histogram showing the percentage of fibers with the indicated numbers of separate fragments in the synaptic contact. The most substantial change occurs between 18 and 22+ months (a combination of ages 22-26 months), as the number of junctions with 10 or more fragments increases from $\sim 25$ to $80 \%$. Number of animals/number of fibers are as follows:P21, 2/121;P42, 2/94; P70, 2/62;P140, 1/51; postnatal 11 months, 2/68; postnatal 18 months, 1/52; postnatal 22-26 months, 4/141.

Table 1. Morphological features of sternomastoid NMJs in aged mice

\begin{tabular}{lcc}
\hline Feature & Frequency & \% of NMJs \\
\hline Fragmentation & $118 / 142$ & 83.1 \\
Dim AChR patches & $7 / 132$ & 5.3 \\
Aneural AChR patches & $20 / 132$ & 15.2 \\
Preterminal axonal swelling & $18 / 125$ & 14.4 \\
Polyneuronal innervation & $9 / 139$ & 6.5 \\
Terminal sprouts & $7 / 135$ & 5.2
\end{tabular}

Sternomastoid muscles from four animals 22-26 months of age were analyzed for features previously shown to occur at aging NMJs (Valdez et al., 2010). An endplate was considered fragmented if it had 10 or more discrete AChR-rich segments/islands. The $142 \mathrm{NMJs}$ had an average of $15.1 \pm 0.5$ fragments. There appeared to be no gender-based disparity for fragmentation (83.3\% of NMJs in males vs $83.0 \%$ of junctions in females had 10 or more fragments).

We observed that most of the NMJs in the oldest animals had fragmented AChRs and varicose nerve terminals (Fig. 1), features of aging previously reported (Valdez et al., 2010). Younger junctions most commonly had smooth, continuous synaptic gutters filled with AChRs and nerve terminals that were smooth and continuous (Fig. 1). Some of the junctions in even the oldest animals we examined had this "young-appearing" phenotype. The incidence of fragmented junctions (defined in our case as having 10 or more fragments of AChRs) increased dramatically to $>80 \%$ of the fibers by 22 months of age as the frequency of the young-appearing junctions declined (Fig. 2). Clearly, events occur that change the basic morphology of these junctions and these events appear to accelerate with age.

We also saw some of the other signs of aging reported by Valdez et al. (2010) (including multiple axonal profiles that appeared to enter the junction, axons containing preterminal swellings, sprouts, and $\operatorname{dim}$ AChR islands that had been abandoned by the nerve) (Table 1), but we saw no signs of denervation. We could produce signs of denervation if the muscles were treated with agents designed to preserve mRNA (Ambion RNAlater), but these same signs of denervation (weakening of the fluorescence of the motor axons to barely detectable levels as well as the physical displacement of some presynaptic structures from junctions during the preparation of thin layers of fibers for microscopy) were present in younger muscles subjected to this same treatment. We 


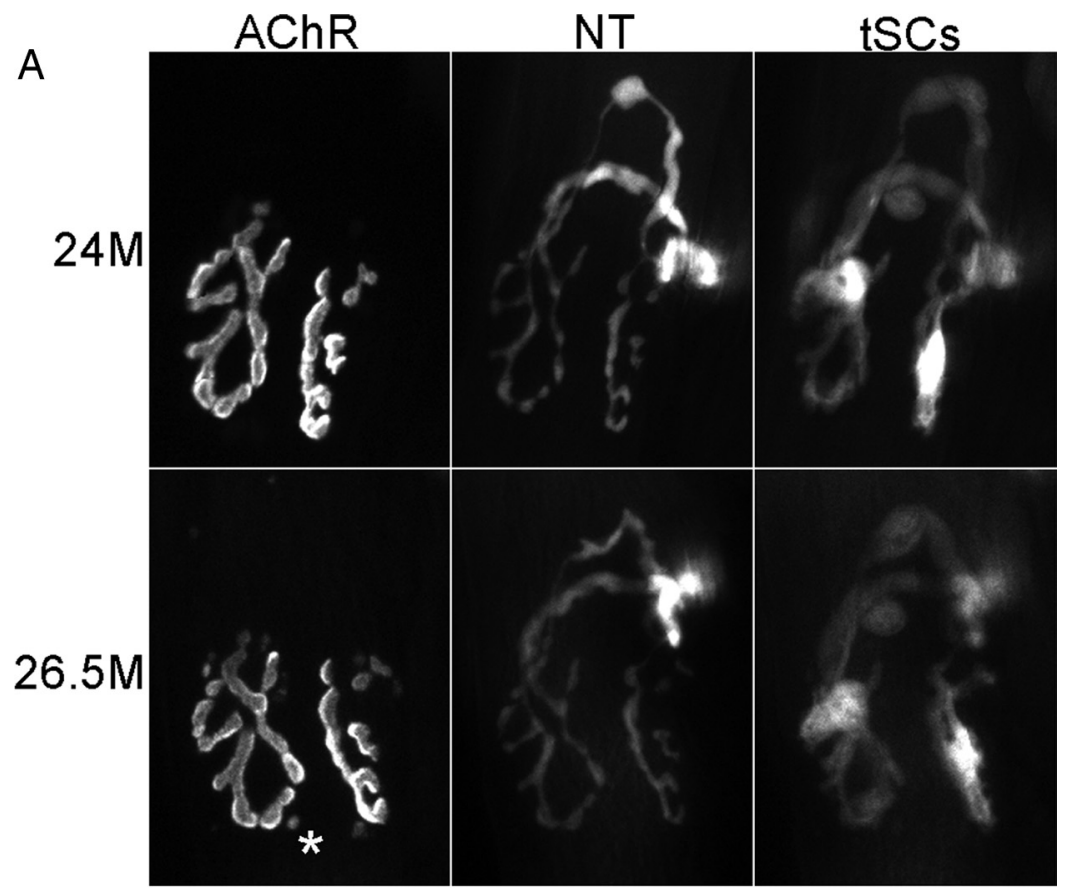

B
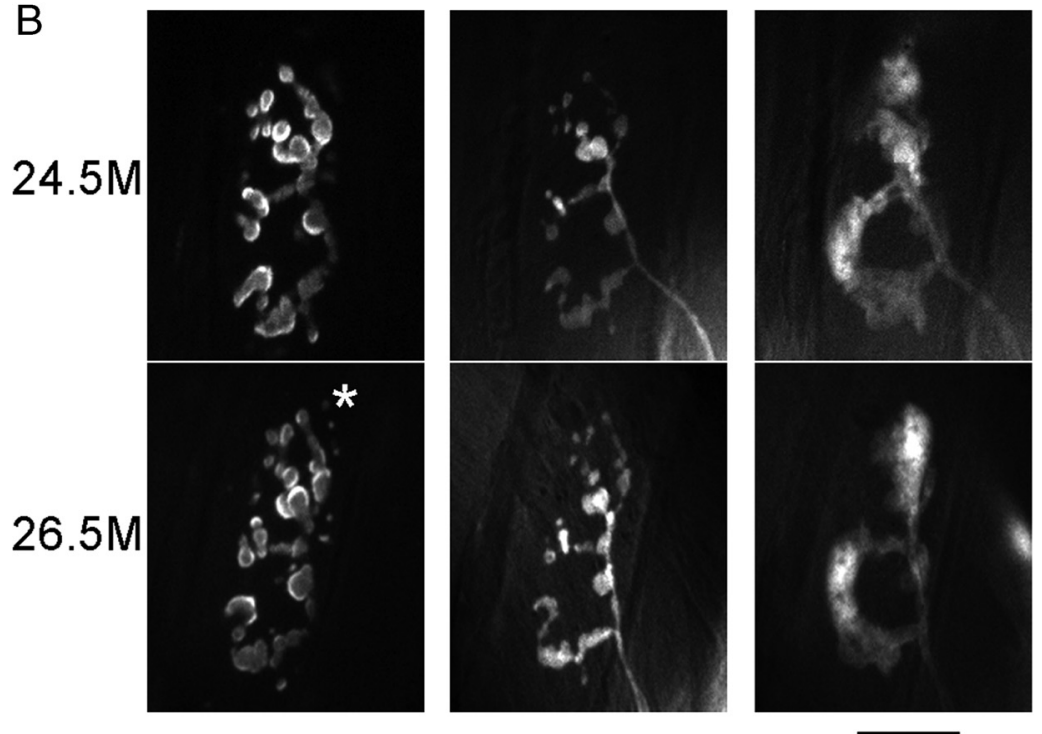

Figure 3. Most NMJs in aging muscles, regardless of whether they have the young or the old phenotype, are stable over time. Images were obtained over the indicated time intervals from the same NMJs. Labeling is as described in Figure 1. An image of AChRs was made during the second imaging of each NMJ (bottom row in each panel) before any additional Alexa 647 bungarotoxin was applied. This image therefore shows the receptors persisting from the previous imaging session. Images of AChRs here and in Figure 4 were captured and are presented at the same camera gain and exposure time so that the intensity differences represent those seen in the microscope. $A$, Young-appearing junction that showed no change and persistent, strong labeling of AChRs from the first imaging session. An additional four images collected between 24 and 26.5 months showed no change. $\boldsymbol{B}$, Fragmented, old-appearing junction that showed no change and persistent strong labeling of AChRs from the previous imaging session. An additional three images between 24.5 and 26.5 showed no change. The asterisks in the two images mark one of several examples where AChRs, labeled in the prior imaging session, appear as spots of label that are now internal to the fiber (Akaaboune et al., 1999). Scale bar, $20 \mu \mathrm{m}$.

did not, however, examine the hindlimb muscles used in a previous study (Valdez et al., 2010) in which death of motor neurons has been consistently reported.

Repeated, vital imaging shows most junctions (youngappearing and old-appearing) change very little over time To examine how individual NMJs in aging mice change over time, we used mice identical with those described above and im- aged the NMJs in the living sternomastoid muscle of the anesthetized individuals under a wide-field fluorescence microscope. The CFP fluorescence revealed the motor axons, and the GFP fluorescence, the Schwann cells. In addition, a nonblocking dose of fluorochrome-conjugated (Alexa 647) bungarotoxin was used to label the AChRs in the postsynaptic muscle membrane. The junctions appeared like those reported above: some were young appearing, but the majority were the fragmented, aged phenotype.

We conducted repeated vital imaging expecting to document the changes that occurred as each animal aged. We typically imaged at 2 week intervals, although the panels exhibited in Figures 3 and 4 were collected at longer intervals. We used the unique morphology of each junction to identify and reimage the same junctions previously imaged. In total, we imaged a set of 32 junctions multiple times in five animals starting at 16-24 months and extending until these animals reached 20.5-26.5 months. We imaged these 32 junctions 4-15 different times for a total length of time that extended from 2 to 6 months. The changes we observed in the structure of these junctions are presented below according to their morphology at the time of the initial observation. We found that most junctions exhibited only minor changes, if any, over many imaging sessions, a surprising finding in view of the expectation of a slow, gradual change.

Of the 32 junctions selected for imaging, 11 were young appearing upon acquiring the first image. As described above, these had mostly smooth, continuous nerve terminals apposed to long stretches of receptors that were unfragmented (Fig. 3A). With one exception (see below), these junctions never changed over the time of our observations: there were no alterations in the nerve terminal, its apposition to AChRs, nor the pretzel pattern of AChRs in the muscle fiber membrane. The labeling of the AChRs obtained with Alexa 647 bungarotoxin applied at the time of the first observation remained strong on subsequent observations, although it decreased from its initial intensity because of the slow, normal turnover of AChRs (Akaaboune et al., 1999). Moreover, reapplication of a new, low dose of Alexa 647 bungarotoxin during a subsequent im aging session labeled the same pretzel pattern seen previously, suggesting a uniform turnover and replacement of these AChRs. Thus, there appear to be a set of young-appearing junctions in these aging animals that, like those reported in younger animals (Balice-Gordon and Lichtman, 1990), are quite stable. 
The remaining 21 junctions in the vital imaging sessions had varicose nerve terminals and AChRs that were fragmented into a number of small islands at the time of the first observation. As these junctions are the ones that appear to be aged, we attempted, in some of the experiments, to bias our sampling toward such junctions, believing that they might be the ones that were changing. In total, 18 of these 21 junctions were mostly stable in their morphology, like the young-appearing junctions described above. For the most part, the varicosities in their terminals changed little in appearance or apposition to islands of AChRs (Fig. 3B). The bungarotoxin labeling from a previous imaging session remained strong, indicating a slow turnover of these receptors; reapplication of bungarotoxin gave a strong labeling that mirrored that of the prior labeling, showing creation of no new receptor sites and no sites that were turning over (with the exceptions to be described next). Despite the picture of overall stability, there were a few junctions in this set that showed minor losses or additions of islands of receptor that corresponded with minor losses or additions of nerve terminal. [For examples of such changes, see the study by Li and Thompson (2011), their Fig. 7.] On occasion, some of these receptor islands were observed to dim in their labeling intensity relative to the remainder of the synaptic site before their disappearance. However, these events were low in frequency and it would be impossible for events like these occurring at the observed frequencies to produce an aged junction from a young-appearing junction, even over many weeks. Moreover, these types of changes were not observed in the young-appearing junctions but only in those that were already fragmented. We will argue below that these small changes are the fallout of other events that more profoundly and immediately alter junctions. That the great majority of junctions did not change argues against the hypothesis that aging produces slow, gradual change in junctions.

\section{Some junctions in aging animals}

undergo a sudden, profound change in their morphology ending with a fragmented, aged appearance In contrast to the stability of the 10 smooth junctions documented above, one smooth junction underwent a sudden transformation between two imaging sessions (Fig. $4 A$ ) so that its nerve terminal became varicose, a feature typical of an aged junction. These changes occurred between two imaging sessions (i.e., over the course of 2 weeks). Not only were the changes in the nerve terminal obvious, but the labeling of AChRs with bunga-
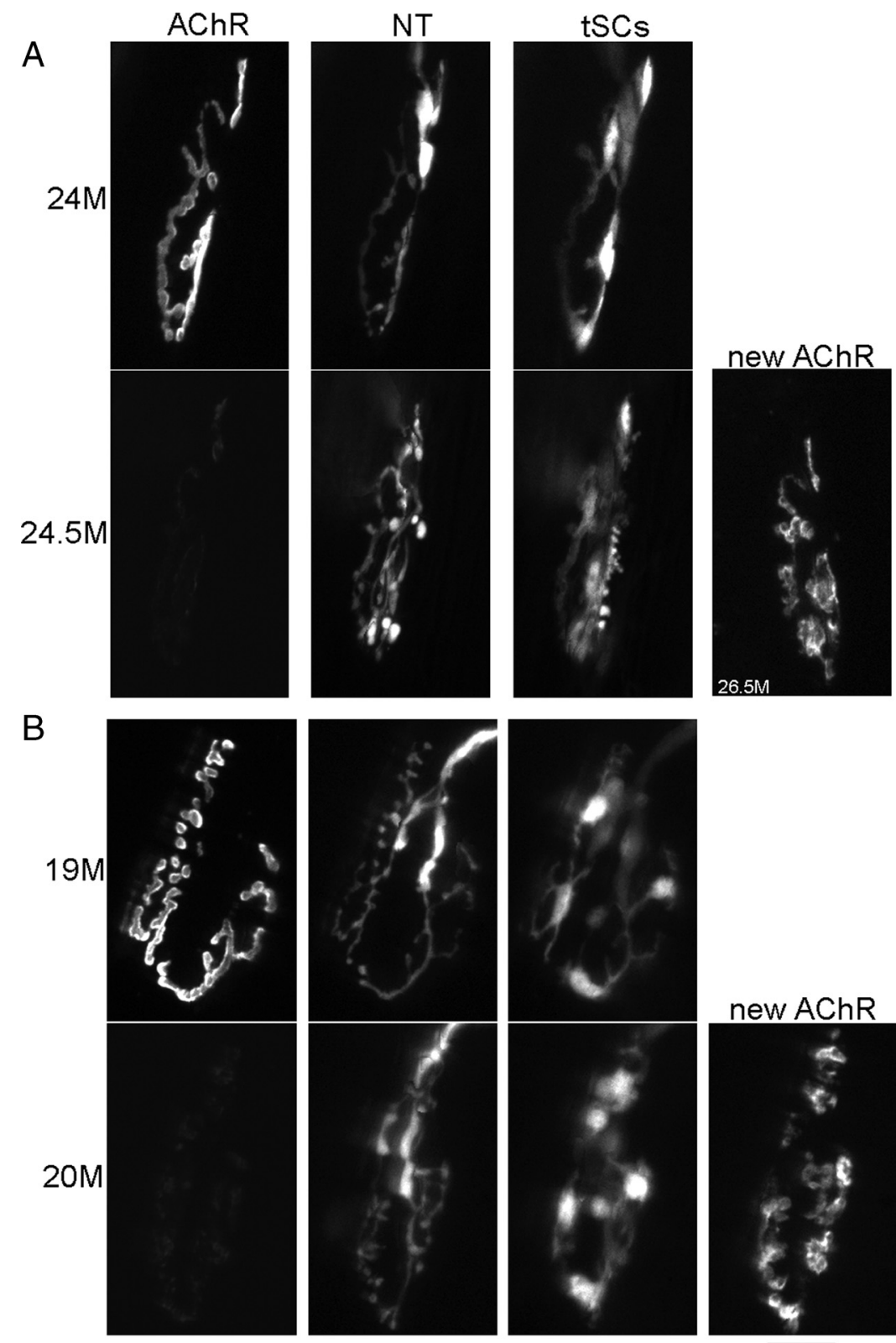

Figure 4. A few NMJs in aging animals exhibit a sudden change in morphology occurring over a very short time period associated with a loss of AChRs throughout the synapse. Labels are described in legends of Figures 1 and 3 . In the bottom row in each panel, there is an additional image displaced to the right. In these cases, the AChR labeling (first image in the row) was very dim, showing that most of these receptors had turned over during the time intervening between this image and the previous one. In these cases, reapplication of new bungarotoxin revealed the receptors that were newly synthesized and inserted (images labeled "new AChR"). A, Young-appearing junction that showed a loss of the first labeling of AChRs, insertion of significant new receptors, and a fragmentation of the AChRs that became more apparent with time as the fiber grew and expanded (rightmost image). In this case, the nerve terminal becomes varicose. $\boldsymbol{B}$, Fragmented, old-appearing junction that showed a loss of the first labeling of AChRs, insertion of significant amounts of new receptors and additional fragmentation of the AChRs. This loss occurred between the 11 th and 12 th image in a sequence beginning at 16 months of age. Scale bar, $20 \mu \mathrm{m}$.

rotoxin was altered as well. The bungarotoxin labeling of AChRs seen in the imaging session immediately before this change had almost vanished and could be detected only by increased gain of the CCD camera. This suggests, given the strong binding between bungarotoxin and AChRs, that the AChRs that had been previously labeled had mostly turned over. Such a turnover is known to occur when the sarcolemma of the muscle fiber itself disappears during segmental necrosis (i.e., as the portion of the muscle fiber beneath the synapse is damaged and degenerates) (Jir- 
manová and Thesleff, 1972; Couteaux et al., 1988; Rich and Lichtman, 1989). Reapplication of the Alexa 647 bungarotoxin in our experiments gave a strong labeling, suggesting newly synthesized AChRs had appeared, but the receptors were now present in fragmented islands. Such newly synthesized receptors are known to appear when the fiber segment regenerates and contacts the old synaptic basal lamina or is contacted by the nerve terminal that remains behind (McMahan and Slater, 1984; Rich and Lichtman, 1989). Thus, the changes seen in these aging NMJs mimic those seen in experiments in which the postsynaptic muscle fiber is deliberately damaged, degenerates, is replaced by muscle satellite cells that form a new fiber segment, and is then innervated by the nerve terminal (Rich and Lichtman, 1989; Li and Thompson, 2011). We will make the argument below that this is what is happening in aging muscle.

The remaining 3 of 21 junctions that, at the first imaging session, were already fragmented and appeared aged, exhibited a sudden instability, just like the one young-appearing junction reported immediately above. They underwent a sudden change between imaging sessions in which the labeling of their AChRs from the previous imaging session became weak and required high gain of the CCD camera to be seen (Fig. 4B). Similarly, when new bungarotoxin was applied, a strong labeling of only some of the islands labeled in the previous imaging session was seen; the remaining islands labeled with the new bungarotoxin were apparently new. Again, the loss of the original label and appearance of new receptors labeled by reapplication of bungarotoxin argues that these junctions resided on a muscle fiber segment that had degenerated and regenerated.

\section{Application of two different fluorescent bungarotoxins at different times expands observations of the events of aging and shows degeneration and regeneration events in aging muscle fibers}

Our vital observations suggested a general, overall stability of individual NMJs punctuated by sudden changes in a seemingly random subset of them. However, since these sudden events are relatively rare and since the vital imaging method samples by necessity only a few NMJs, our sample size is very small. Furthermore, in many cases our selection of junctions for imaging was biased. In some cases, we biased the samples toward youngappearing junctions, and in other cases, toward aged junctions, always expecting to document slow, ongoing change. We therefore sought a means of obtaining observations that would sample many more fibers in the muscle in an unbiased fashion. The vital imaging experiments suggested that the loss of AChRs seen in the greatly diminished bungarotoxin labeling would be a method for identifying fibers that had recently undergone such change. We reasoned we could vitally label almost all the junctions in the sternomastoid muscle by a single application of a low, nonblocking concentration of a first-color bungarotoxin (Alexa 647) and allow the animal to fully recover from the procedure without imaging individual junctions. After 2 weeks had elapsed, the animal could be killed, the muscle fixed, and the AChRs labeled with a second-color bungarotoxin (rhodamine). We term this method below the two-color method; it copies a method used by Akaaboune et al. (1999) to study AChR turnover. We expected a decline in the labeling intensity of the first color in every junction because of slow turnover of receptors. However, if the fiber had degenerated and regenerated during this period, then we would expect the labeling intensity of these junctions to be much weaker than those of the surrounding fibers, as most of the AChRs would have turned over. This is the meaning of the "strong," "weak" description of the first
Table 2. Types of junctions encountered in aged muscles as a result of the two-color bungarotoxin experiments

\begin{tabular}{llllll}
\hline & \multicolumn{2}{l}{ Status of AChR labeling/status of AChR plaque } \\
\cline { 2 - 3 } & \multicolumn{2}{l}{ First weak, second strong } & & First strong, second strong \\
\cline { 2 - 3 } \cline { 5 - 6 } & AChR fragmented & AChR smooth & & AChR fragmented & AChR smooth \\
\hline Central nuclei present & $6^{a}$ & 0 & & $7^{b}$ & 0 \\
Central nuclei absent & 0 & 0 & & $133^{c}$ & $25^{d}$ \\
\hline
\end{tabular}

Data are from 171 NMJs examined in three muscles at 25-32 months of age, 2 weeks following vital application of first-color bungarotoxin and at the time of application of second color. Numerical entries are given superscripts that indicate their interpretation as follows.

${ }^{a}$ Six (4\%) NMJs on fibers that experienced a degeneration/regeneration episode in the postsynaptic segment in the 2 week period; all have central nuclei.

${ }^{b}$ Seven (4\%) NMJs on fibers that experienced a degeneration/regeneration episode in the postsynaptic segment prior to start of the 2 week period but not so long ago that central nuclei have completed their migration to the periphery; central nuclei are present.

'One hundred thirty-three (78\%) NMJs on fibers that experienced a degeneration/regeneration episode in the postsynaptic segment more than $\sim 30 \mathrm{~d}$ before; these have no central nuclei.

${ }^{d}$ Twenty-five (15\%) NMJs that appear "young" and are located on fibers that, in contrast to all the NMJs above, have never experienced degeneration/regeneration of the postsynaptic segment of muscle fiber; these have no central nuclei.

labeling that follows. DAPI was also applied to label nuclei. We therefore examined a large sample of individual junctions by static imaging of the fixed muscle.

The vital imaging experiments make several predictions. Because they would not have undergone fiber degeneration, the smooth, young-appearing junctions encountered after the 2 weeks should have a strong first-color label and a strong secondcolor label; none of these fibers should have a weak labeling with the first color. All junctions with weak first-color label, strong second-color label would have fragmented AChRs and varicose nerve terminals. Moreover, the NMJs in this latter category should be present on muscle fibers that had recently regenerated. Muscle fibers or fiber segments that have recently undergone regeneration should have central nuclei (i.e., a string of nuclei located in the center of the fiber that form by the fusion of the myoblasts participating in the muscle repair) (Karpati and Molnar, 2008). Last, we would also predict that there would be junctions that had become fragmented long in advance of the application of the first-color label and that these would show strong first-color, strong second-color label.

These predictions were all verified with the two-color method by the observations of 171 muscle fibers in three muscles from animals 25-32 months of age, examined over the course of 2 weeks (Table 2).

Approximately $15 \%$ of the fibers had smooth synaptic gutters $(<10$ fragments) and nerve terminals that had smooth branches; none of these NMJs had weak first-color labeling, and none of these had central nuclei when the confocal microscope was used to examine the position of DAPI-labeled nuclei in $z$-stacks (Fig. $5 A$ ). We interpret these to be junctions that had never undergone a degeneration/regeneration event that involved the segment of their muscle fiber where the synapse was located.

Approximately $4 \%$ of the fibers labeled and examined 2 weeks later with this two-color method had weak first label, strong second label, fragmented AChRs, and varicose nerve terminals (Fig. $5 B)$. In these cases, central nuclei were present in the region of the NMJ (Fig. 5B). Unfortunately, since we have no image of these junctions before the supposed degeneration event, we do not know their original status (i.e., whether they were youngappearing or aged); however, from the vital imaging experiments, we presume that there are at least some junctions in this group that were young appearing. However, overall, $\sim 85 \%$ of the muscle fibers were fragmented and had varicose nerve terminals. 


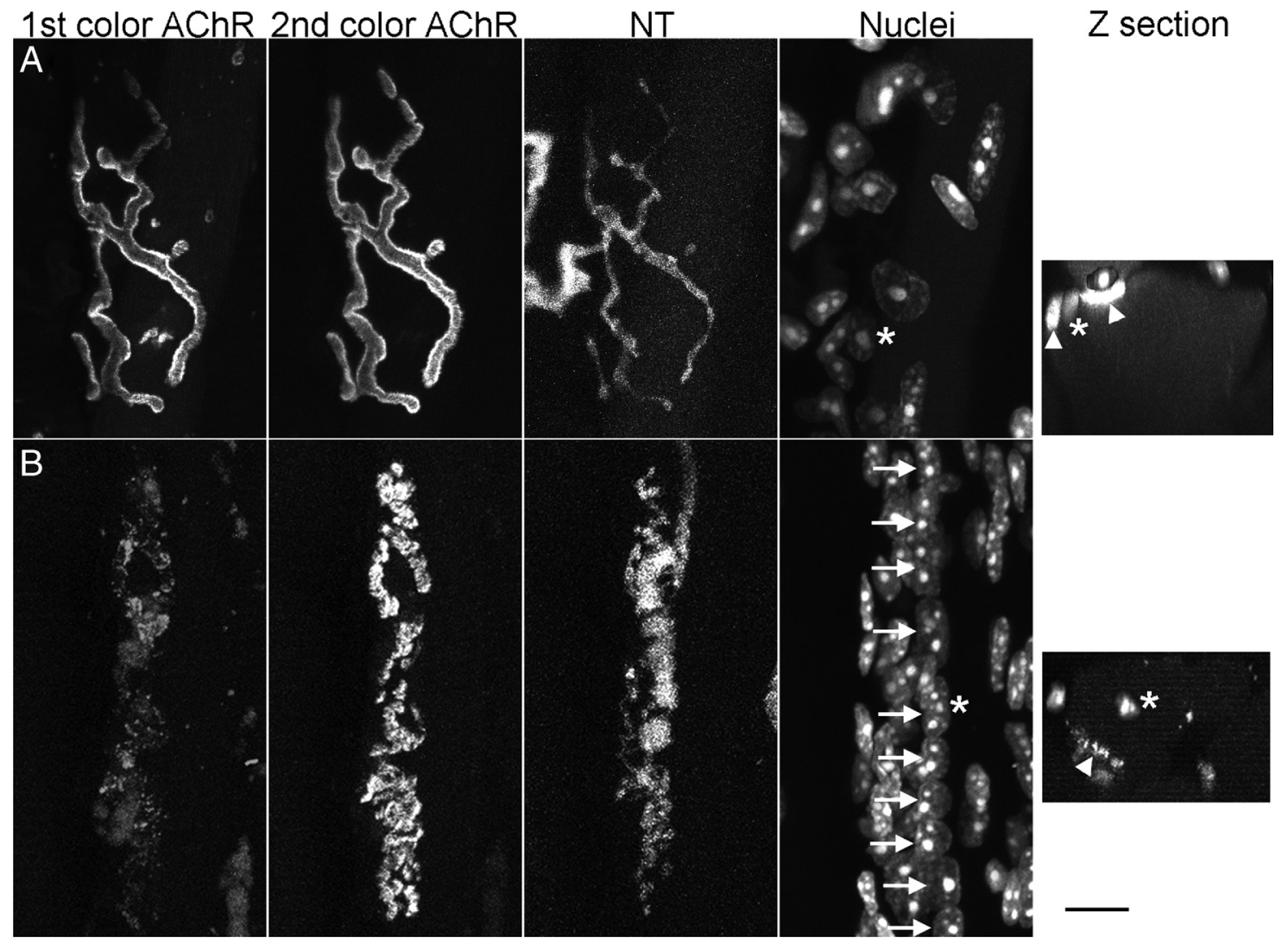

Figure 5. A two-color method of bungarotoxin labeling of AChRs combined with nuclear labeling identifies fibers where the muscle segment underneath the NMJ has degenerated and regenerated and altered the synapse. Alexa 647 bungarotoxin (first color) was applied vitally at a nonsaturating concentration to the sternomastoid muscle. Two weeks later, the muscle was dissected, fixed, labeled with rhodamine- bungarotoxin (second color) and with DAPI, and imaged by confocal microscopy. Confocal $z$-slices (far right images) were prepared in each case and the contrast and brightness manipulated so that outline of the muscle fiber could be ascertained as well as a fiber nucleus (asterisk) and AChRs (arrowheads). $\boldsymbol{A}, \boldsymbol{B}$, Components of each of two junctions from the same 25.5-month-old muscle: first-color AChRs, second-color AChRs, nerve terminals, and nuclei (DAPI). $\boldsymbol{A}$, Young-appearing junction with strong first-color labeling whose fiber nuclei are at the periphery. $\boldsymbol{B}$, Junction whose first color labeling is much weaker, whose fiber has a string of central nuclei (arrows) and whose AChRs have become fragmented. Each asterisk in the en face images identifies the same nucleus shown with an asterisk in the z-section. The terms "strong" and "weak" in reference to the first color label are relative to each other, as explained in the text. Scale bar, $10 \mu \mathrm{m}$.

With the exception of the $4 \%$ of junctions just described, none of these fibers had weak first label, strong second label, suggesting the event that created them had occurred in advance of the application of the first color. Interestingly, not only did all the fibers with newly fragmented junctions (those with weak first color and strong second color) have central nuclei underneath the NMJ, $4 \%$ of the older fragmented junctions (those with strong first color, strong second color) did as well. That the majority of the fragmented junctions did not have fibers with central nuclei in the synaptic region suggests that either these fragmented junctions were not generated as a result of degeneration and regeneration or that the central nuclei, with time, migrate to the periphery in regenerated fibers, a subject of some controversy in the literature (Jirmanová and Thesleff, 1972; Duchen et al., 1974; Schmalbruch, 1976; Couteaux et al., 1988; Karpati et al., 1988; Rich and Lichtman, 1989; Terada et al., 2010).

\section{Central nuclei are a transient phenomena in single mouse muscle fibers regenerating after segmental necrosis}

To investigate the fate of central nuclei in regenerating muscle fibers, we deliberately damaged individual muscle fibers underneath NMJs in young adult mice by irradiating the muscle fiber on each side of, but not within, the junction with a laser microbeam (van Mier and Lichtman, 1994; Li and Thompson, 2011). This ablation was done vitally with fibers whose NMJs (and those of adjoining unablated fibers) were labeled with a low dose of rhodamine-bungarotoxin to complement the fluorescent protein labeling of their nerve terminals and SCs. We then examined these very same muscle fibers and their junctions $6-133 \mathrm{~d}$ after the ablation, by killing the animal, fixing the muscle, and labeling nuclei with DAPI. After 6-10 d, as reported previously (Li and Thompson, 2011), the NMJs on ablated muscle fibers were found to have AChRs that were beginning to fragment and nerve terminals that were becoming varicose (Fig. 6). Of all the fibers ablated in our previous study, we observed that all of their NMJs ( 32 of 32) had fragmented receptors at times $>14 \mathrm{~d}$ following ablation. However, junctions on the adjacent, nonablated control fibers had the expected morphology for young junctions in animals of this age. All the ablated muscle fibers had long strings of central nuclei present in the fiber in the vicinity of the NMJs, but the adjacent control fibers had nuclei at the periphery (Fig. 6). These results demonstrate the effectiveness of the ablation procedure. Interestingly, the nuclei in the junction itself soon assumed a position at the periphery underneath the endplate, as no central nuclei were seen directly beneath the junction $30 \mathrm{~d}$ after ablation. Junctions examined $30 \mathrm{~d}$ after ablation $(n=8$ junctions) still had a few central nuclei just outside the endplate, but most of the nuclei had a peripheral location. By $133 \mathrm{~d}$ (the next time examined; $n=4$ junctions), there were no central nuclei. These observations suggest that the fragmented junctions 


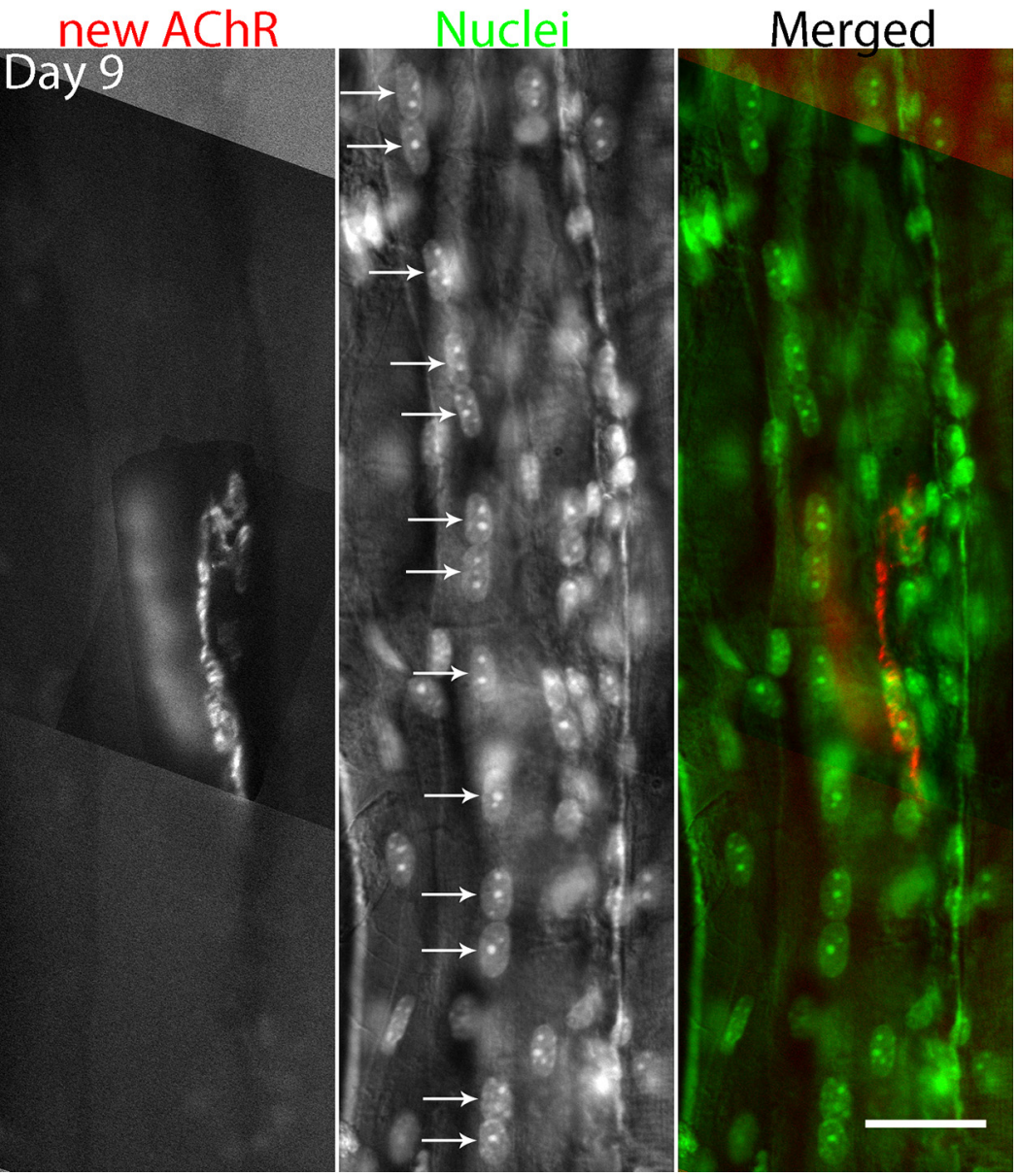

Figure 6. Deliberate ablation of a muscle fiber with a laser microbeam applied to each side of the NMJ leads to degeneration of the underlying muscle fiber, followed by regeneration, and NMJ fragmentation. The junction is labeled with bungarotoxin and the nuclei with DAPI. In this case, $9 \mathrm{~d}$ after ablation, a string of central nuclei remains in the vicinity of the junction. Similar experiments with longer survival times after ablation show that the central nuclei eventually disappear following regeneration (see text). The central location of these nuclei could be confirmed by confocal imaging (as described in Fig. 5). Scale bar, $30 \mu \mathrm{m}$.

without central nuclei seen in the two-color bungarotoxin experiments had nonetheless undergone degeneration and regeneration. If one estimates that $8 \%$ of the NMJs undergo a degenerative event in the postsynaptic muscle fiber every month, then a steady state of $\sim 8 \%$ of the fibers would be expected to have such central nuclei in this location provided these nuclei have a lifetime in this location of approximately a month.

\section{Regeneration of fiber segments is a common feature of aging muscle}

Previous observations suggest a high fraction of fibers in aging muscle have central nuclei. For example, Valdez et al. (2010) in their study of aged mice showed that $12-18 \%$ of the fibers in the lower hindlimb had central nuclei. This would underestimate the total involvement of fibers in the muscle if short segments in each fiber, rather than the entire fiber, were affected. Indeed, muscle whole mounts suggest that this is the case (see below). How can a high frequency of degeneration/regeneration be reconciled with a lower incidence of synaptic change?

During the course of the two-color experiments, we made incidental observations of strings of central nuclei in nine muscle fibers that were located entirely outside the endplate band. When the NMJs on these fibers were examined, they all had strong first-color bungarotoxin staining and moreover three of them were of the young-appearing morphology. These observations show that damage/ regeneration events that do not involve the muscle segment underneath the NMJ do not alter the morphology of the junction. Thus, synaptic change appears to occur only when the degeneration/regeneration event involves the segment of the fiber underneath the synapse.

We examined the distribution of fiber segments with central nuclei along the length of aging muscle fibers (Fig. 7). For this purpose, a thin, sheet-like muscle, the levator auris, from a 27-month-old mouse was examined in a confocal microscope after labeling nuclei with DAPI, NMJs with bungarotoxin, and the muscle fiber membranes with an antibody directed against the protein dystrophin. A number of fibers (1727) were followed along one-half their length $(\sim 1500 \mu \mathrm{m})$ from one tendon to the center of the muscle and its NMJs. These fibers contained a total of 42 segments of centrally located nuclei, ranging from one nucleus in length to seven nuclei in a chain. Some of the fibers had more than one segment with central nuclei. This observation shows that, if these central nuclei result from fiber damage, damage events are frequent in aging muscle and appear at apparently random locations along the length of fibers. The total frequency of these events along the muscle is much higher than that at the junction, likely because each NMJ occupies only a small fraction of the length of each fiber.

\section{Discussion}

This study provides strong evidence that the major morphological features of aging neuromuscular junctions can be accounted for by changes that occur in the postsynaptic muscle fiber leading to its necrosis/degeneration and regeneration. Instead of finding that junctions in aging animals undergo gradual change, the vital imaging shows that they are mostly stable except during the renewal of the muscle fiber segment at the synapse. It is likely that this connection between the junction and fiber necrosis has gone unrecognized because the events occur rapidly and leave, except for the changes in the junction, no signs that can be easily observed. The central nuclei that occur during regeneration are transient. Moreover, central nuclei occur throughout aging muscles and predict a synaptic change only when they are present at the synapse. At least one previous study pointed to the similarities in the morphology of aged junctions and those resulting from fiber degeneration and regeneration (Rich and Lichtman, 1989), but a firm connection, to our knowledge, has not been made. The results of this study seem entirely compatible with previous studies linking changes in junctional morphology to diseases in which muscle fiber damage occurs (Lyons and Slater, 1991).

Our study suggests that segments of muscle fibers degenerate and regenerate and produce a change in the morphology of the aging NMJ. However, at present, the only purported persistent manifestation of these events is this junctional change itself; this 


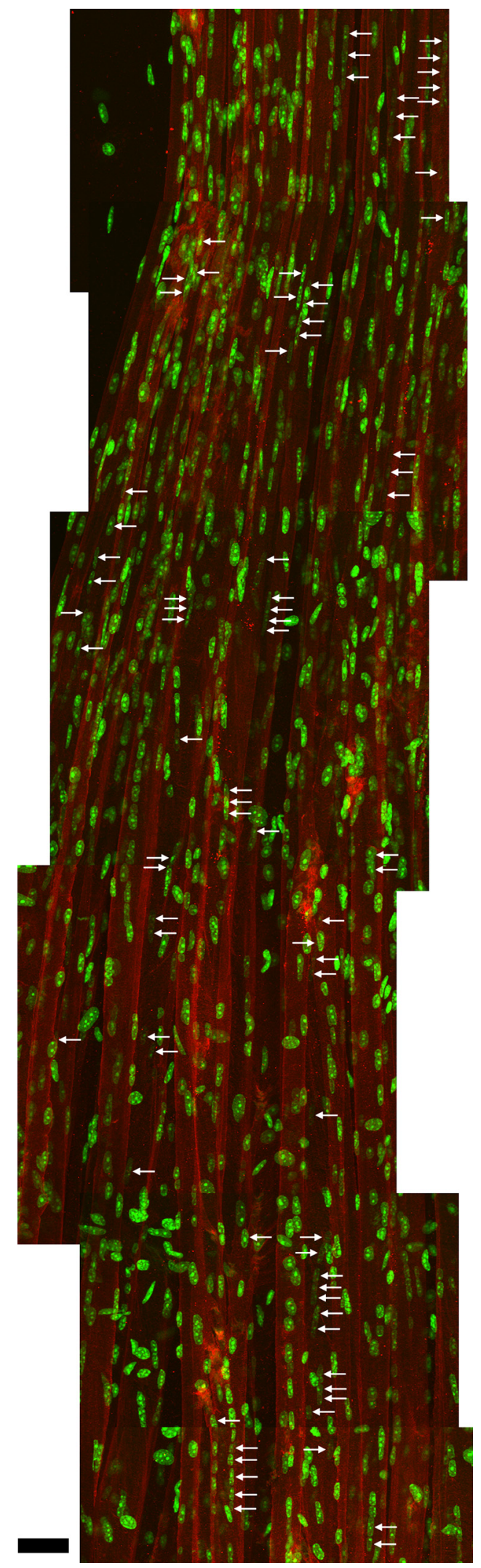

Figure 7. The location of central nuclei along muscle fibers suggests that degeneration/ regeneration events are quite frequent in older animals and are apparently randomly distributed. A levator auris muscle from a 27-month-old mouse was labeled in whole mount for dystrophin to identify the outlines of the muscle fibers, with bungarotoxin to identify the NMJs, and with DAPI to identify nuclei. A montage of maximum projections of overlapping confocal stacks containing 17-27 fibers at one edge of the muscle was made. The total extent examined is a limitation to our study. We believe that the vital imaging and the two-color method provide strong evidence that most NMJs are stable but a small percentage of them change following degeneration/regeneration of the segment underneath the junction. Since the changes in the junctions seem to be permanent (Rich and Lichtman, 1989; Li and Thompson, 2011), we believe it is possible to account for the number of changed junctions by injuries that occur randomly along muscle fibers over an appreciable time interval during aging. However, at the moment, we do not know many things about this injury, whether it is truly random, the length of the necrotic segment, and even whether the kinetics of fiber injury, fiber replacement, and junctional change resemble those that follow deliberate injury to muscle fibers with a laser microbeam. These issues await further investigation.

Our results do not explain all phenomena associated with aging junctions. For example, denervation is reported to occur in limb muscles (Valdez et al., 2010). For unknown reasons, denervation appears absent from the sternomastoid muscle. Nonetheless, the sternomastoid junctions and those in the limb appear to undergo the same morphological changes, and central nuclei are prominent in both. Our study suggests why central nuclei would be an expected feature of muscles undergoing such synaptic changes.

The results presented here also do not imply that fiber degeneration and regeneration is the only way junctions can become fragmented. There are a number of genetic manipulations in mice that have been shown to produce such a morphology without any sign of muscle fiber turnover (cf. Fox et al., 2007; Banks et al., 2009). The mechanisms leading to fragmentation in these cases remain unclear, and some of them could be held in common with the changes following fiber degeneration/regeneration during aging. While it is clear fiber necrosis and regeneration even in young muscles is a stimulus for fragmentation, the molecular mechanisms by which nerve terminals become varicose and fragment the junctions are not clear.

The morphological changes seen in aging are also not limited to older animals. The mdx mouse model of muscular dystrophy begins to fragment junctions at $\sim 1$ month of age. Although rare, fully fragmented junctions can be found in 1- to 2-month-old sternomastoid muscles of normal mice (data not shown), suggesting that the transformation can occur over relatively short time periods even in young mice.

Previous studies of the physiology of aging muscles suggest that there are decrements in the performance of these synapses (Kelly, 1978; Balice-Gordon, 1997), and it will be interesting to attempt to correlate physiological changes with those in the morphology. It is presently unclear exactly how the morphological changes in the synapses are orchestrated, although a recent study from this laboratory (Li and Thompson, 2011) and an older study (Rich and Lichtman, 1989) suggest that this involves growth of the nerve terminal into novel contacts stimulated by the regeneration of the muscle fiber. Ablation of muscle fiber segments at the $\mathrm{NMJ}$ in young adult mice shows that the nerve terminal com-

was $1500 \mu \mathrm{m}$ along the length of the fibers from one tendon (bottom) to the band of NMJs (top); only $1000 \mu \mathrm{m}$ is shown here. Central nuclei, some of which are in chains within individual fibers, are identified with arrows. The central location of the nuclei within the fibers was ascertained from examination of confocal $z$-sections. A total of 42 fiber segments with central nuclei was found of varying length (the longest, $67 \mu \mathrm{m}$ ), suggesting that fiber damage is a frequent event in aging muscle. Variation in the number of central nuclei is likely explained by the severity of the damage event and/or the time elapsed since the injury, as nuclei move to the periphery of the fibers. Scale bar, $30 \mu \mathrm{m}$. 
monly remains intact over the old synaptic basal lamina until the fiber begins to regenerate ( $\mathrm{Li}$ and Thompson, 2011). The subsequent, aging-like changes in the morphology of the synapse are clearly initiated by this postsynaptic damage. We believe that similar events underlie neuromuscular synaptic changes prominently associated with aging. It is presently unclear whether the incidence of damage events in the old muscles increases with age, although previous studies have reported that aging muscles (as well as muscles weakened by disease) are more susceptible to experimental damage (Brooks and Faulkner, 1990; Petrof et al., 1993) and aging muscles reportedly regenerate less well (Rando, 2006). It is clear from our study that the junctional segments of fibers that have been injured once can be injured again and it is possible that multiple injuries produce more striking changes, perhaps eventually including denervation of fibers and sprouting (Valdez et al., 2010). Since damaging (i.e., eccentric) contractions in vivo could involve activation of particular motor units, damage may be unequally distributed across muscles or preferentially distributed to certain types of fibers. Finally, treatments or procedures that alter the aging of these synapses (e.g., those achieved by caloric restriction and exercise) (Valdez et al., 2010) may change the susceptibility of muscle fibers to injury. It is intriguing that caloric restriction is reported not only to reduce the aging of junctions but also to reduce dramatically the frequency of fibers with central nuclei.

\section{References}

Akaaboune M, Culican SM, Turney SG, Lichtman JW (1999) Rapid and reversible effects of activity on acetylcholine receptor density at the neuromuscular junction in vivo. Science 286:503-507.

Balice-Gordon RJ (1997) Age-related changes in neuromuscular innervation. Muscle Nerve Suppl 5:S83-S87.

Balice-Gordon RJ, Lichtman JW (1990) In vivo visualization of the growth of pre- and postsynaptic elements of neuromuscular junctions in the mouse. J Neurosci 10:894-908.

Balice-Gordon RJ, Breedlove SM, Bernstein S, Lichtman JW (1990) Neuromuscular junctions shrink and expand as muscle fiber size is manipulated: in vivo observations in the androgen-sensitive bulbocavernosus muscle of mice. J Neurosci 10:2660-2671.

Banks GB, Chamberlain JS, Froehner SC (2009) Truncated dystrophins can influence neuromuscular synapse structure. Mol Cell Neurosci 40:433-441.

Brooks SV, Faulkner JA (1990) Contraction-induced injury: recovery of skeletal muscles in young and old mice. Am J Physiol 258:C436-C442.

Courtney J, Steinbach JH (1981) Age changes in neuromuscular junction morphology and acetylcholine receptor distribution on rat skeletal muscle fibres. J Physiol 320:435-447.

Couteaux R, Mira JC, d'Albis A (1988) Regeneration of muscles after cardiotoxin injury. I. Cytological aspects. Biol Cell 62:171-182.

Duchen LW, Excell BJ, Patel R, Smith B (1974) Changes in motor end-plates resulting from muscle fibre necrosis and regeneration. A light and electron microscopic study of the effects of the depolarizing fraction (cardiotoxin) of Dendroaspis jamesoni venom. J Neurol Sci 21:391-417.

Feng G, Mellor RH, Bernstein M, Keller-Peck C, Nguyen QT, Wallace M, Nerbonne JM, Lichtman JW, Sanes JR (2000) Imaging neuronal subsets in transgenic mice expressing multiple spectral variants of GFP. Neuron 28:41-51.

Fox MA, Sanes JR, Borza DB, Eswarakumar VP, Fässler R, Hudson BG, John SW, Ninomiya Y, Pedchenko V, Pfaff SL, Rheault MN, Sado Y, Segal Y, Werle MJ, Umemori H (2007) Distinct target-derived signals organize formation, maturation, and maintenance of motor nerve terminals. Cell 129:179-193.

Gutmann E, Hanzlíková V, Vysokocil F (1971) Age changes in cross striated muscle of the rat. J Physiol 216:331-343.

Jirmanová I, Thesleff S (1972) Ultrastructural study of experimental muscle degeneration and regeneration in the adult rat. Z Zellforsch Mikrosk Anat 131:77-97.

Karpati G, Molnar MJ (2008) Muscle fibre regeneration in human skeletal muscle diseases. In: Skeletal muscle repair and regeneration (Schiaffino S, Partridge T, eds), pp 199-215. Dordrecht, The Netherlands: Springer.

Karpati G, Carpenter S, Prescott S (1988) Small-caliber skeletal muscle fibers do not suffer necrosis in $\mathrm{mdx}$ mouse dystrophy. Muscle Nerve 11:795-803.

Kelly SS (1978) The effect of age on neuromuscular transmission. J Physiol 274:51-62.

Li Y, Thompson WJ (2011) Nerve terminal growth remodels neuromuscular synapses in mice following regeneration of the postsynaptic muscle fiber. J Neurosci 31:13128-13136.

Lichtman JW, Magrassi L, Purves D (1987) Visualization of neuromuscular junctions over periods of several months in living mice. J Neurosci $7: 1215-1222$

Lingle CJ, Steinbach JH (1988) Neuromuscular blocking agents. Int Anesthesiol Clin 26:288-301.

Lyons PR, Slater CR (1991) Structure and function of the neuromuscular junction in young adult mdx mice. J Neurocytol 20:969-981.

Marques MJ, Conchello JA, Lichtman JW (2000) From plaque to pretzel: fold formation and acetylcholine receptor loss at the developing neuromuscular junction. J Neurosci 20:3663-3675.

McMahan UJ, Slater CR (1984) The influence of basal lamina on the accumulation of acetylcholine receptors at synaptic sites in regenerating muscle. J Cell Biol 98:1453-1473.

Petrof BJ, Shrager JB, Stedman HH, Kelly AM, Sweeney HL (1993) Dystrophin protects the sarcolemma from stresses developed during muscle contraction. Proc Natl Acad Sci U S A 90:3710-3714.

Rando TA (2006) Stem cells, ageing and the quest for immortality. Nature 441:1080-1086.

Rich M, Lichtman JW (1989) Motor nerve terminal loss from degenerating muscle fibers. Neuron 3:677-688.

Schmalbruch H (1976) The morphology of regeneration of skeletal muscles in the rat. Tissue Cell 8:673-692.

Terada M, Lan YB, Kawano F, Ohira T, Higo Y, Nakai N, Imaizumi K, Ogura A, Nishimoto N, Adachi Y, Ohira Y (2010) Myonucleus-related properties in soleus muscle fibers of $\mathrm{mdx}$ mice. Cells Tissues Organs 191:248-259.

Valdez G, Tapia JC, Kang H, Clemenson GD Jr, Gage FH, Lichtman JW, Sanes JR (2010) Attenuation of age-related changes in mouse neuromuscular synapses by caloric restriction and exercise. Proc Natl Acad Sci U S A 107:14863-14868.

van Mier P, Lichtman JW (1994) Regenerating muscle fibers induce directional sprouting from nearby nerve terminals: studies in living mice. J Neurosci 14:5672-5686.

van Mier P, Balice-Gordon R, Lichtman J (1994) Synaptic plasticity studied in vivo using vital dyes, lasers, and computer-assisted fluorescence microscopy. Neuroprotocols 5:91-101.

Walsh MK, Lichtman JW (2003) In vivo time-lapse imaging of synaptic takeover associated with naturally occurring synapse elimination. Neuron 37:67-73.

Wood SJ, Slater CR (2001) Safety factor at the neuromuscular junction. Prog Neurobiol 64:393-429.

Zuo Y, Lubischer JL, Kang H, Tian L, Mikesh M, Marks A, Scofield VL, Maika S, Newman C, Krieg P, Thompson WJ (2004) Fluorescent proteins expressed in mouse transgenic lines mark subsets of glia, neurons, macrophages, and dendritic cells for vital examination. J Neurosci 24:10999-11009. 